\title{
Biomarkers in Irritable Bowel Syndrome: Biological Rationale and Diagnostic Value
}

\author{
Radislav Nakov ${ }^{a}$ Violeta Snegarova $^{\mathrm{b}}$ Desislava Dimitrova-Yurukova ${ }^{c}$ \\ Tsvetelina Velikova $^{d}$ \\ ${ }^{a}$ Clinic of Gastroenterology, Tsaritsa Yoanna University Hospital, Medical University of Sofia, Sofia, Bulgaria; \\ ${ }^{\mathrm{b}}$ Department of Hygiene and Epidemiology, Medical University of Varna, Varna, Bulgaria; 'Department of \\ Gastroenterology, Pulmed University Hospital, Plovdiv, Bulgaria; ${ }^{d}$ Department of Clinical Immunology, University \\ Hospital Lozenetz, Sofia University St. Kliment Ohridski, Sofia, Bulgaria
}

\author{
Keywords \\ Irritable bowel syndrome - Biomarkers - Cytokines · \\ Fecal calprotectin - Short-chain fatty acids - Microbiome · \\ MicroRNA
}

\begin{abstract}
Background: Patients with irritable bowel syndrome (IBS) usually suffer from nonspecific and overlapping signs that hamper the diagnostic process. In line with this, biomarkers specific for IBS could be of great benefit for diagnosing and managing patients. In IBS, the need is for apparent distinguishing features linked to the disease that improve diagnosis, differentiate from other organic diseases, and discriminate between IBS subtypes. Summary: Some biomarkers are associated with a possible pathophysiologic mechanism of IBS; others are used for differentiating IBS from non-IBS patients. Implementation of IBS biomarkers in everyday clinical practice is critical for early diagnosis and treatment. However, our knowledge about their efficient use is still scarce. Key Messages: This review discusses the biomarkers implemented for IBS diagnosis and management, such as blood (serum), fecal, immunological, related to the microbiome, microRNAs, and some promising novel biomarkers associ-
\end{abstract}

ated with imaging and psychological features of the disease. We focus on the most commonly studied and validated biomarkers and their biological rationale, diagnostic, and clinical value.

(c) 2021 S. Karger AG, Basel

\section{Introduction}

A wide variety of symptoms and manifestations make the definite diagnosis of irritable bowel syndrome (IBS) a continued challenge. The differential diagnosis is so extensive that it may take years to establish the correct diagnosis. Clinicians should exclude inflammatory bowel disease (IBD), celiac disease, microscopic colitis, lactose intolerance, fructose intolerance, colon cancer, gastrointestinal (GI) infections, and transthyretin amyloidosis [1] and the symptoms of hyperparathyroidism, hypothyroidism, and neuroendocrine tumors [2].

Currently, the syndrome can only be diagnosed based on accurate identification of the main clinical symptoms using modified criteria from Rome IV, 2016 (Fig. 1) [3]. According to Rome IV criteria, IBS is defined as a functional gastrointestinal disorder in which recurrent ab- 
Diagnosis of irritable bowel syndrome according to Rome IV criteria for asults and children

Adults

Recurrent abdominal pain with onset at least six months prior to diagnosis, associated with two or more of the following,

at least one day per week in the last three monts:

- Related to defecation

- Associated with a change in frequency of stool

- Associated with a change in form (appeareance) of stool

\section{Children}

Abdominal pain at least four days per months, for at least two

months before diagnosis with one or more of the following:

- Related to defecation

- Associated with a change in frequency of stool

- Associated with a change in form (appeareance) of stool

Fig. 1. Rome IV criteria for diagnosis of IBS in both adults and children (adapted from Drossman [3]). IBS, irritable bowel syndrome.

dominal pain is correlated with a change in bowel habits or defecation. Symptoms should be present during the last 3 months, and the symptom onset should have started at least 6 months before diagnosis. Abdominal bloating/distension and disordered bowel habits (i.e., constipation, diarrhea, or a mix of constipation and diarrhea) are some of the commonly observed symptoms [4]. Making an early diagnosis based on a clinical evaluation of symptoms while limiting the use of investigations is crucial for the best IBS management.

However, exhaustive investigation to exclude all organic pathology is still made in many IBS patients. Some IBS patients suffer from nonspecific and overlapping signs that cannot be associated with a specific diagnosis. In line with this, biomarkers specific for IBS could be of great benefit in the clinician's armamentarium in diagnosing IBS.

Typically, a biomarker can be simply described as a defining characteristic, measured as an indicator of normal biological processes, pathogenic processes, or responses to an exposure or intervention [5]. Biomarkers can be derived from molecular, histologic, radiographic, or physiologic characteristics of the disease. Moreover, several subtypes of biomarkers have been defined according to their putative applications.

Notably, a single biomarker may meet multiple criteria for different uses. Still, it is essential to develop evidence for each definition. In the case of IBS, while definitions for biomarkers may overlap, they also have apparent dis- tinguishing features that specify particular uses. For example, diagnostic biomarkers, monitoring biomarkers, pharmacodynamic/response biomarkers, predictive biomarkers, prognostic biomarkers, and others can be implemented for IBS [6].

Indeed, there are several types of biomarkers for IBS aiming to improve diagnosis, to differentiate from other organic diseases and to discriminate between IBS subtypes. Some biomarkers are associated with a possible pathophysiologic mechanism of IBS, and others are used for differentiating IBS from non-IBS patients [7].

Nevertheless, an ideal biomarker should fulfill the following criteria: high sensitivity and specificity, simple to use, reproducibility, low interobserver variability, and affordable and acceptable for the patient [8]. Implementation of IBS biomarkers in everyday clinical practice is critical for early diagnosis and treatment of this functional gastrointestinal disorder. However, our knowledge about their efficient use is still scarce.

In this review, we make an overview of the biomarkers discussed for IBS diagnosis and management, such as blood (serum), fecal, immunological, microbiome, microRNAs, and some promising novel biomarkers associated with imaging and psychological features of the disease. We focus on the most common, studied, and validated biomarkers and their biological rationale, diagnostic, and clinical value (Table 1).

\section{Blood Biomarkers for IBS Management}

\section{Serum-Based Panels of Biomarkers for IBS}

Some blood-based biomarkers can provide valuable information for patients who have IBS. Although blood work can offer diagnostic clues, there are no molecules present in the blood currently recognized unique to IBS. Furthermore, although the diagnosis of IBS depends on the clinical symptoms, serological markers for diagnosis can also be utilized.

For this reason, Lembo et al. [9] conducted a study to investigate a combination of 10 blood biomarkers. These 10 biomarkers were interleukin-1ß (IL-1ß), growth-related oncogene-a, brain-derived neurotrophic factor (BDNF), anti-Saccharomyces cerevisiae antibody (ASCA IgA), antibody against CBir1, anti-tissue transglutaminase ( $\mathrm{tTG}$ ), tumor necrosis factor (TNF)-like weak inducer of apoptosis, anti-neutrophil cytoplasmic antibody, tissue inhibitor of metalloproteinase-1 (TIMP-1), and neutrophil gelatinase-associated lipocalin (NGAL). The authors demonstrated that this panel of biomarkers had a 
Table 1. Biomarkers for IBS diagnosis and management

\begin{tabular}{|c|c|c|c|c|c|c|c|}
\hline Type & Biomarkers & $\begin{array}{l}\text { Control } \\
\text { group }\end{array}$ & $\begin{array}{l}\text { Sensitivity, } \\
\%\end{array}$ & $\begin{array}{l}\text { Specificity, } \\
\%\end{array}$ & PLR & NLR & AUC \\
\hline \multirow[t]{4}{*}{$\begin{array}{l}\text { Blood } \\
\text { biomarkers }\end{array}$} & $\begin{array}{l}\text { A panel of } 10 \text { biomarkers (IL-1ß, GRO-a, BDNF, ASCA IgA, } \\
\text { anti-CBir1, tTG, TWEAK, ANCA, TIMP-1, and NGAL) [9] }\end{array}$ & Non-IBS & 50.0 & 88.0 & 4.17 & 0.57 & 0.76 \\
\hline & $\begin{array}{l}\text { A panel of } 34 \text { serologic (including histamine, tryptase, serotonin, and } \\
\text { substance P), gene expression markers, and psychological } \\
\text { measurements [14] }\end{array}$ & $\mathrm{HC}$ & 81.0 & 64.0 & 2.25 & 0.30 & 0.81 \\
\hline & Anti-CdtB antibodies [22] & Non-IBS & 43.7 & 91.6 & 5.20 & 0.61 & 0.81 \\
\hline & Anti-vinculin antibodies [22] & Non-IBS & 32.6 & 83.8 & 2.01 & 0.80 & 0.62 \\
\hline $\begin{array}{l}\text { Immune } \\
\text { activation } \\
\text { biomarkers }\end{array}$ & $\begin{array}{l}\text { Inflammatory cytokines - IL- } 6 \text { and its receptor, IL- } 8 \text {, and IL- } 1 \beta \text {, } \\
\text { TNF- } \alpha\end{array}$ & $\mathrm{n} / \mathrm{a}$ & & & & & \\
\hline \multirow{4}{*}{$\begin{array}{l}\text { Fecal } \\
\text { biomarkers }\end{array}$} & Fecal SCFA [38] & $\mathrm{HC}$ & 92.0 & 72.0 & 3.29 & 0.11 & 0.89 \\
\hline & Secretogranin II [39] & $\mathrm{HC}$ & 80.0 & 79.0 & 3.81 & 0.25 & 0.86 \\
\hline & $\begin{array}{l}\text { A panel of } 8 \text { biomarkers (IL- } 1 \beta, \text { IL-6, IL-12p70, TNF- } \alpha, \text { CgA, HBD2, } \\
\text { caproate, and calprotectin) [35] }\end{array}$ & $\mathrm{HC}$ & 88.1 & 86.5 & 6.53 & 0.14 & 0.89 \\
\hline & Volatile organic metabolites [42] & $\mathrm{HC}$ & 90.0 & 80.0 & 4.50 & 0.13 & 0.94 \\
\hline Microbiome & No specific microbial biomarker & $\mathrm{n} / \mathrm{a}$ & & & & & \\
\hline $\begin{array}{l}\text { MicroRNA } \\
\text { biomarkers }\end{array}$ & miRNA-199, miRNA-150, and miRNA-342-3p & $\mathrm{n} / \mathrm{a}$ & & & & & \\
\hline
\end{tabular}

IBS, irritable bowel syndrome; ANCA, anti-neutrophil cytoplasmic antibody; anti-CBir1, antibody against CBir1; anti-CdtB, anti-cytolethal distending toxin B; ASCA, anti-Saccharomyces cerevisiae antibody; BDNF, brain-derived neurotrophic factor; CgA, chromogranin A; GRO-a, growth-related oncogene-a; HBD2, human beta-defensin-2; IBD, inflammatory bowel disease; IL, interleukin; n/a, not applicable; NGAL, neutrophil gelatinase-associated lipocalin; NLR, negative likelihood ratio; PLR, positive likelihood ratio; SCFA, short-chain fatty acids; TIMP-1, tissue inhibitor of metalloproteinase-1; TNF- $\alpha$, tumor necrosis factor alpha; tTG, anti-tissue transglutaminase; TWEAK, tumor necrosis factor-like weak inducer of apoptosis.

positive predictive value of $81 \%$, a negative predictive value of $64 \%$, and an overall accuracy of $70 \%$. Moreover, the investigators concluded that the proposed diagnostic panel of 10 biomarkers could differentiate between IBS and non-IBS GI disorders.

The cytokine IL-1ß was chosen because of its central role in inflammatory diseases such as IBD. It is well known that glucocorticoids released during stress have a significant downregulatory effect on IL-1ß [10]. However, the role of IL-1B in IBS remains uncertain, and further studies are required to investigate this potential biomarker and validate it.

Another immune factor under investigation is the growth-related oncogene. It is thought that this might be responsible for the tissue injury in IBS patients [9]. This chemokine is associated with chemotactic migration and activation of neutrophils.

Irritable Bowel Syndrome Biomarkers
$\mathrm{BDNF}$ is another potential biomarker under investigation; it is a member of the neurotrophin family and is thought to play a crucial role in many chronic pain conditions. It has been previously noted that patients with IBS have significant increases in total nerve fibers and damage on the mucosal nerve fibers (e.g., swollen mitochondria and nerve axons) with increased BDNF, and this correlated with the abdominal pain scores $[11,12]$. This, therefore, shows potential as a biomarker for IBS patients subtype with abdominal pain.

The last biomarker in the panel was NGAL, a $25-\mathrm{kDa}$ protein. NGAL participates in various roles in viscera, including molecular transportation and GI mucosal regeneration [13], with a possible role in IBS-related disruptions in the barrier function.

Another study involving 168 IBS subjects (60 IBS-C, 57 IBS-D, and 51 IBS-M) and 76 healthy volunteers aimed 
to differentiate the performance of a combination of 34 serologic and gene expression markers and psychological measurements. A total of 10 serological markers were added to the original 10-biomarker panel, including histamine, tryptase, serotonin, and substance $\mathrm{P}$, together with 14 gene expression markers from analysis of differentially expressed genes in IBS and healthy people, including CBFA2T2, CCDC147, and ZNF326 [14].

Results show that the panel registered a sensitivity of $81 \%$ and a specificity of $64 \%$, together with good discrimination between IBS subtypes, the best being for the subtype IBS-C versus IBS-D. However, one of the study's limitations is that healthy volunteers were characterized as adults without any illness, active infection, or significant medical condition. Still, there was no reference to functional symptoms. Another drawback to the study is that no comparison with other organic diseases was provided. According to the investigators, their biomarker panel would best discriminate IBS from organic GI disorders [14].

\section{Differentiating IBS from IBD}

Notably, there are well-established serological markers that help differentiate IBS from IBD. These include ASCA, which facilitates the differential diagnosis of Crohn's disease (CD) and ulcerative colitis (UC), predominantly in the disease's early stages. The serum concentration of ASCA is considerably higher in patients with CD than in those with UC [15]. Thus, ASCA can be employed in differentiating organic disease from IBS. Furthermore, a study examining the levels of ASCA in $\mathrm{CD}$ and murine colitis concludes that the propensity to produce ASCA in a subgroup of CD patients is mostly genetically predetermined, as evidenced by their stability and lack of correlation with clinical disease activity parameters.

The other autoantibodies that can be used in distinguishing IBS from IBD are the anti-neutrophil cytoplasmic antibody. They target antigens present in neutrophils and are positive in $50-80 \%$ of the UC patients [16].

Tissue inhibitors of metalloproteinases (TIMPs), on the other hand, are endogenous protein regulators of the matrix metalloproteinase family. Altered TIMP activity can lead to disruption of the intestinal barrier and excessive immune response [17]. TIMPs have been shown to play a role in tissue degradation and remodeling. Indeed, in patients with IBD, tissue remodeling is observed due to chronic inflammation. Serum levels of TIMP-1 and TIMP-4 have been known to be related to IBD [18], but their role in patients with IBS has not yet been studied well.
A study published in 2018 followed the effects of biopsy supernatants on human and guinea pig submucous neurons with neuroimaging techniques, utilizing 7 healthy controls, 20 IBS, and 12 UC patients. The researchers differentiated by proteome analysis several expressed proteins such as proteases that were cleaving proteinase-activated receptors (PARs). PARs are proteins that are involved in protein degradation and the regulation of cell functions. The subtype PAR2 receptor is highly expressed in colonic epithelial cells and is involved in secretion, visceral sensation, inflammation, and motility $[19,20]$. It is well known that the GI tract is exposed to proteases produced by the stomach, small intestine, inflammatory cells (mast cells), and bacteria.

A total of 204 proteins have been identified after a proteome analysis of the supernatants. Out of all investigated proteins, 17 proteases were differently expressed between IBS, UC, and healthy controls. Of these, significantly more abundant in IBS than healthy and UC supernatants were chymotrypsin $\mathrm{C}$, an unspecified isoform of complement $\mathrm{C} 3$, proteasome subunit type beta-2, and proteases elastase $3 \mathrm{a}$. The results indicated that the UC supernatants activated enteric neurons through protease-dependent mechanisms but without PAR1 involvement. Moreover, PAR1 antagonist SCHE79797 prevents nerve activation by IBS supernatants. The combination of cathepsin $\mathrm{L}$, elastase $3 \mathrm{a}$, and proteasome alpha subunit- 4 reveals 98\% accuracy in differentiating between IBS and healthy people. Thus, the authors conclude that proteases signaling through neuronal PAR1 may be used as IBS biomarkers [21].

In a recent study that included 2,681 subjects $(2,375$ IBS-D patients, 43 healthy subjects, 121 celiac patients, and 142 IBD patients), the researchers assessed the sensitivity of anti-cytolethal distending toxin B (anti-CdtB) antibodies and anti-vinculin antibodies. The results indicated that anti-CdtB demonstrated specificity and sensitivity 91.6 and $43.7 \%$ and for anti-vinculin were 83.8 and $32.6 \%$, respectively, concerning IBS-D. These results confirm that anti-CdtB and anti-vinculin antibodies are elevated in IBS-D compared to non-IBS subjects [22].

\section{Differentiating Celiac Disease from IBS}

Anti-tTG is the gold standard in the serologic diagnosis of celiac disease [19]. However, a study published in 2019 , involving 44 women and 26 men with IBS, showed that $7.1 \%$ of participants were positive for IgA and IgG anti-tTG (3 of them had diarrhea-predominant IBS [IBSD] and 2 constipation-predominant IBS [IBS-C]). Patients diagnosed with mixed bowel habits (IBS-M) did
Nakov/Snegarova/Dimitrova-Yurukova/ Velikova 
not give a positive result. Moreover, in patients with IBS$\mathrm{D}$, the possibility of celiac disease should be ruled out [23].

\section{Biomarkers for IBS Related to Immune Activation}

Immune activation is a known phenomenon in some patients with IBS. IBS has long been viewed as a neurological condition resulting from modifications in the brain-gut axis. However, immunological modifications are frequently reported in IBS patients, compatible with the hypothesis that there is a chronic low-grade immune activation. The mediators released by immune cells act to either depress or increase the activity of GI nerves. The release of a number of these mediators corresponds to symptoms of IBS, highlighting the significance of immune and nervous system connections [24, 25].

Critical players in gut reactions are eosinophils and mast cells. Eosinophils usually are related to allergic responses; however, no changes in numbers of eosinophils in intestinal biopsies, blood, or the in situ levels of eosinophil cationic protein are seen in IBS [26]. In line with this, several studies have linked eosinophil numbers with functional dyspepsia, a functional GI disorder associated with upper abdominal discomfort and disordered motility $[27,28]$. However, little is known about the effects eosinophil-derived mediators have on GI nerves. Mast cells and their mediators from colonic mucosal biopsies may also activate sensory afferent neurons.

Several inflammatory cytokines are elevated in serum and secreted by peripheral blood mononuclear cells in IBS patients, including IL- 6 and its receptor, IL-8, and IL$1 \beta$, compared to healthy controls; however, this is not observed in all IBS patients. There are conflicting results for TNF- $\alpha$, with reports of increased and decreased levels documented in IBS $[29,30]$.

Interestingly, there was a correlation between cytokines and symptoms; thus, lipopolysaccharide-induced TNF-a production in peripheral blood mononuclear cells was associated significantly with anxiety $(r=0.59, p<$ 0.001 ) [29]. However, a subgroup of IBS patients with elevated serum cytokine levels (IL-6, IL-8, and TNF- $\alpha$ ) and decreased mucosal expression of IL-10 was generally associated with protection from immune activation. Those IBS patients with immune activation had a shorter transit time compared to those without immune activation. The TNF- $a$ level was positively correlated with looser stool consistency and increased rectal sensitivity. At the same time, those with more frequent bowel movements had higher IL-6 levels [31].

Irritable Bowel Syndrome Biomarkers
At the same time, an increase in activated B lymphocytes and plasma cells and increased IgG levels in the jejunal fluid in IBS-D patients compared to healthy controls had also been shown. The immune activation is usually paralleled by mucosal mRNA upregulation of heavy immunoglobulin chains, acting as biological markers of humoral activity. The latter correlated with the consistency and number of stools per day in these patients [32].

Despite many decades of promising evidence implicating the immune system's role in IBS pathogenesis, its degree of association with clinical symptoms remains controversial. Many questions remain. Foremost is causation; what drives the immune activation - is it centrally or peripherally mediated, or a combination of both? IBS patients frequently copresent with extraintestinal and psychological comorbidities, including anxiety and depression, which correlate with altered immune function, and emotional stimuli such as chronic stress are known to alter immune function [2]. There is a need for further investigation of the immune mechanisms that might be involved in IBS development.

\section{Fecal Biomarkers in Diagnosis and Management of IBS}

Fecal biomarkers have become among the most significant biomarkers of GI diseases that allow noninvasive testing. In general, they have been developed to reflect inflammation of the intestinal mucosa. In line with this, their primary role is to identify IBD and, therefore, to exclude IBS.

\section{Fecal Calprotectin}

Fecal calprotectin (FC) is a small calcium- and zincbinding protein that is found in abundance in neutrophilic granulocytes as well as in monocytes and macrophages [33]. FC is the most frequently studied marker for intestinal inflammation.

A study by Waugh et al. $[25,34]$ demonstrated that FC had a sensitivity of $93 \%$ and a specificity of $94 \%$ at a cutoff level of $50 \mu \mathrm{g} / \mathrm{g}$ in differentiating IBS from IBD. In most cases, negative calprotectin rules out IBD, thereby sparing most people with IBS from having to have invasive investigations, such as colonoscopy.

A recent study with 196 IBS patients and 160 healthy controls without GI symptoms demonstrated that a panel of 8 biomarkers (IL-1 $\beta$, IL-6, IL-12p70, TNF- $\alpha$, fecal chromogranin A [CgA], human beta-defensin-2, caproate, and FC) had a sensitivity of $88.1 \%$ and a specificity of $86.5 \%$ in discriminating IBS subjects from healthy people. These popu- 
lations were obtained from the Maastricht IBS cohort. However, validation of this biomarker panel for the discrimination between organic GI disorders was not performed [35].

\section{Fecal Short-Chain Fatty Acids}

Fecal short-chain fatty acids (SCFAs) are derived from nondigestible carbohydrates through gut microbial fermentation [36]. They include acetate, propionate, butyrate, valerate, and caproate. Products of microbial fermentation of nondigested oligosaccharides in the colon are selected as gut intraluminal metabolic activity indicators. Since SCFAs have been associated with multiple pathological and physiological mechanisms in humans, for example, modulation of inflammation, satiety, and carcinogenesis, and are an essential energy source for colonocytes [37], they are promising biomarkers for different conditions.

To date, SCFAs have only been studied in small population (25 IBS subjects and 25 healthy controls) aimed to diagnose IBS by measuring fecal SCFA. This study noted that differences in the values of butyric and propionic acid had the best diagnostic properties, with a sensitivity of $92 \%$ and a specificity of $72 \%$ at a cutoff level $>0.015$ $\mathrm{mmol} / \mathrm{L}$ [38].

\section{Granins, Chromogranins, and Secretogranins}

Granins are proteins distributed ubiquitously in vesicles of secretory cells of the enteric, endocrine, and immune system. It is thought that they may serve as markers for the activity of the enteric neuroendocrine system [39]. $\mathrm{CgA}$ was selected as a marker of intestinal neuroendocrine cell activity. This peptide is produced by enterochromaffin cells and is colocalized in storage granules with serotonin $[39,40]$. Secretogranin (Sg)III, on the other hand, is found in mast cells. Öhman et al. [39] reported that CgA, SgII, and SgIII were elevated in feces samples of 82 IBS patients compared to 29 healthy volunteers. Furthermore, it was demonstrated that an SgII value of $0.16 \mathrm{nmol} / \mathrm{g}$ identified IBS with a sensitivity of $80 \%$, a specificity of $79 \%$, and an AUC of 0.86 on the receiver operating curve.

To date, the role of granins in the pathophysiology of IBS is not completely clear, and the reason why levels of granins are different in IBS subjects has not been understood $[7,41]$. In contrast to the fecal measurements, studies of duodenal, ileal, and colonic mucosa showed reduced densities of CgA immunoreactive cells in IBS compared to healthy controls [41].

\section{Volatile Organic Metabolites}

VOCs are low-molecular-weight metabolic compounds with high vapor pressures and low boiling points, promoting evaporation at ambient temperatures. There is rising evidence that particular VOCs are unique to various disorders. In a study that explored VOM, they recruited 30 patients with IBS-D, 62 with CD, 48 with UC, and 109 healthy controls. In this study, they found 240 VOMs of interest. Univariate analysis showed that esters of SCFA, cyclohexane carboxylic acid, and its ester derivatives were associated with IBS-D, while aldehydes were significantly more abundant in IBD. With 11 key VOMs, the discriminatory model discriminated IBS-D from patients with active $\mathrm{CD}$ and $\mathrm{UC}$ with a sensitivity of 94 and $96 \%$, respectively, and a specificity of 82 and $80 \%$, respectively. Furthermore, IBS-D could be significantly distinguished from the healthy controls (90\% sensitivity and $80 \%$ specificity) [42].

\section{Microbiome Studies in IBS Patients}

The GI tract contains a dense society of commensal bacteria. The relationship between the host and the microbe is immunologically complex, as commensals comprise a balance between beneficial and harmful strains in health. Any disruptions in this homeostasis are associated with disease states $[43,44]$. The gut microbiota is most abundant in the colon, with a switch in strain predominance from Gram-positive aerobes to Gram-negative anaerobes. Therefore, the colon is the primary site of fermentation and subsequent production of organic acids and gases [43].

The microbiome has been studied in IBS with some consistent findings; among these is the demonstration of high Firmicutes and decreased Bacteroides in IBS patients compared to healthy volunteers [43-45]. However, there have been conflicting results with high Actinobacteria in IBS in one study and low Bifidobacterium (Actinobacteria) in other studies [43-45].

Such variable findings may conceivably be attributable to dietary differences in the studied patients. In a recent study, IBS patients (predominantly IBS-D and IBS-M, rather than IBS-C) had a greater Bacteroides enterocyte community than did healthy controls who had more Prevotella species, and the IBS symptom severity score (IBSSSS) increased as the prevalence of Prevotella species decreased [46].

It has been proposed that these microbial alterations cause symptoms in IBS by alteration in cytokine levels. 
Firmicutes and other bacteria in the microbiome produce flagellin, which is postulated to cause an inflammatory response in IBS. A significantly higher serum value of lipopolysaccharide in patients with IBS-D than controls contrasts with the higher level of antibodies to flagellin in patients with IBS (mainly driven by higher levels in IBSD) [47].

Recently, mucosal Brachyspira colonization was described to be significantly more common in IBS-D than in healthy controls, which could be potentially used as a marker for differentiating IBS-D [48]. In summary, there is, at present, no specific microbial biomarker for IBS, and, therefore, there is a limited clinical utility for the use of stool microbial biomarkers for diagnosis or monitoring.

\section{MicroRNA Biomarkers in IBS Pathogenesis}

MicroRNAs (miRNAs) are involved in regulating normal biological functioning processes such as cellular development, differentiation, proliferation, apoptosis, and metabolism. Therefore, dysregulation of miRNA can result in human diseases, including GI disorders [49]. Moreover, many useful biomarkers can be established among miRNAs involved in the pathogenesis of IBS.

miRNAs are short noncoding RNA molecules that play a role in the posttranslational regulation of messenger RNAs. In addition to observations in IBS animal models, there are data on miRNAs from patients with IBS. Thus, miRNA-24 is upregulated in IBS and may aggravate IBS symptoms by inhibiting the serotonin reuptake transporter. Therefore, it may function as a clinically useful biomarker to identify a subset of IBS patients and, potentially, future treatments that can either inhibit miRNA-24 or restore normal serotonin reuptake transporter function [50].

In a study that surveyed the role of miRNAs, Zhou et al. [51] showed that miRNA-29 increases intestinal permeability by reducing claudins and nuclear factor- $\mathrm{kB}-$ repressing factor levels and decreasing glutamine levels. miRNA-29 affects intestinal membrane permeability through its regulation of the glutamate-ammonia ligase gene $[51,52]$. Thus, miRNA-29 could identify a subset of IBS patients with increased membrane permeability who may also benefit from glutamine treatment.

Also, there was a decreased level of colonic miRNA199, which usually decreases visceral pain by inhibiting the signaling of the transient receptor potential vanilloid type 1 . Moreover, this decrease correlated with visceral

Irritable Bowel Syndrome Biomarkers pain in patients with IBS-D [53]. Thus, miRNA-199 may be a potential marker for visceral pain and may be a target for therapy in the future. The other 2 microRNAs, miRNA-150 and miRNA-342-3p, were found to increase in patients with IBS compared to healthy controls [54].

A recent study has reported that microRNA-related serotonin receptor gene expression control with the cisregulatory version affected this regulation and appeared to be related to the female IBS-D [55]. Serotonin receptor type $3(5-\mathrm{HT} 3 \mathrm{E})$ is an essential neurotransmitter in the gut, and abnormal 5-HT3E signaling has been implicated in several functional GI disorders, including IBS. The regulation of miRNA-510 on 5-hydroxytryptamine 3 receptor (5HT3E) expression and the possible association between 5-HT3E single nucleotide polymorphism rs56109847 in IBS-D are exciting findings of this study. The small sample size and study population that is restricted to women are the limitations of this study. However, the serotonin receptors are an exciting potential biomarker.

\section{Future Perspectives}

The use of MRI and diffusion imaging has uncovered differences in brain structure and connectivity between IBS patients and healthy subjects. Brain connectivity differences were also found between genders among IBS patients $[56,57]$. In addition to prefrontal regions, abnormalities in distinct networks in the brain were observed in patients with chronic visceral abdominal pain, including salience, emotional arousal, and sensorimotor networks. Furthermore, therapies such as educational interventions, hypnotherapy, and antidepressants can improve those functional abnormalities shown on brain imaging $[58,59]$. Brain functional imaging offers powerful tools to study important pathophysiological aspects of IBS. However, it is difficult to consider such abnormalities in imaging findings as typical biomarkers. Still, they might be developed further in combination with other markers. However, their high cost and restricted availability limit their clinical and research use significantly. Thus, they are unlikely to serve as routine markers in IBS at the moment.

Psychological assessment was also used as a "marker" of illness in IBS. The addition of psychological measures, such as the Hospital Anxiety and Depression (HAD) scale, the Patient Health Questionnaire 15 (PHQ-15), and the Perceived Stress Scale, to IBS biomarker panels enhanced the ability of the panel to differentiate IBS cases from healthy volunteers, without improving the ability of 
the panels to discriminate between the different subtypes of IBS [60]. Psychological markers are likely not sufficient to diagnose IBS if used alone. Still, they could improve the performance of other biomarkers. When proven in select cases, functional and structural brain dysfunction and psychological disturbances in IBS can be targeted by neuromodulators.

The overarching limitation in the development of biomarkers for IBS is that this is not 1 disease. To establish a biomarker that can identify all patients with IBS is extremely unlikely. Instead, a promising approach is recognizing IBS as multiple diseases with similar symptoms and developing biomarkers to identify the different subgroups to allow targeted therapies. Another limitation is that IBS remains a very heterogeneous group of disorders, and we are not yet great at differentiating them. There is also a vast overlap. This means that until we understand the disease better and the mechanisms that may underpin it, it may be hard to find biomarkers specific to the disease.

\section{Conclusion}

The complex and multifactorial etiology of IBS may mean that a single biomarker that can diagnose IBS with the accuracy required for a clinically useful test cannot be found. To date, symptom-based diagnostic criteria, biomarkers, and psychological markers perform modestly in predicting IBS. Moreover, IBS biomarkers are disappointing due to small study populations and the challenges of ruling out other organic diseases with modest accuracy. In terms of the positive likelihood ratio, the most effective are FC and intestinal permeability markers.
Immune biomarkers have profoundly contributed to our understanding of various aspects of IBS pathophysiology. Moreover, specific immune biomarkers correlate with disease subtypes, making it possible to distinguish the mechanisms underlying the different IBS subgroups. At present, there are several proposed biomarkers (i.e., IL-1 $\beta$, IL-6, IL-12p70, TNF- $\alpha$, CgA, HBD2, calprotectin, and caproate). Still, very few of them have been validated in a diverse group of patients. Even fewer are used now in routine clinical practice. Therefore, combining symptoms with markers appears more effective and may represent the way forward in IBS diagnosis. Biomarkers need to be validated in diverse populations for them to be generalizable.

\section{Conflict of Interest Statement}

The authors have no conflicts of interest to declare.

\section{Funding Sources}

The authors did not receive any funding.

\section{Author Contributions}

R. Nakov had the idea to make the manuscript; R. Nakov, V. Snegarova, D. Dimitrova-Yurukova, and T. Velikova wrote the manuscript and approved the final version of the manuscript. R. Nakov and T. Velikova supervised the whole process.

\section{References}

1 Nakov R, Sarafov S, Gospodinova M, Kirov A, Chamova T, Todorov T, et al. Transthyretin amyloidosis: testing strategies and model for center of excellence support. Clin Chim Acta. 2020;509:228-34.

2 Ford CA, Sperber DA, Corsetti M, Camilleri M. Irritable bowel syndrome. Lancet. 2020; 396(10263):P1675-88.

3 Drossman AD; The Rome IV Committees. History of functional gastrointestinal symptoms and disorders and chronicle of the Rome Foundation. In: Drossman DA, Chang LC, Kellow WJ, Tack J, Whitehead WE, editors. Rome IV functional gastrointestinal disorders: disorders of gut-brain interaction. I. Raleigh, NC: The Rome Foundation; 2016. p. 549-76.
4 Lacy BE, Patel NK. Rome criteria and a diagnostic approach to irritable bowel syndrome. J Clin Med. 2017;6(11):99.

5 FDA-NIH Biomarker Working Group. BEST (Biomarkers, EndpointS, and other Tools) Resource. Silver Spring (MD): Food and Drug Administration (US); Bethesda (MD): National Institutes of Health (US); 2016. Available from: www.ncbi.nlm.nih.gov/books/ NBK326791/ Accessed 2017 Sep 22

6 Califf RM. Biomarker definitions and their applications. Exp Biol Med. 2018;243(3):21321.

$7 \mathrm{Kim} \mathrm{JH}$, Lin E, Pimentel M. Biomarkers of irritable bowel syndrome. The Korean society of neurogastroenterology and motility. J Neurogastroenterol Motil. 2017;23:20-6.
8 Spiller CR. Potential biomarkers. Gastroenterol Clin North Am. 2011;40(1):121-39.

9 Lembo AJ, Neri B, Tolley J, Barken D, Carroll $\mathrm{S}$, Pan $\mathrm{H}$. Use of serum biomarkers in a diagnostic test for irritable bowel syndrome. Aliment Pharmacol Ther. 2009;29(8):834-42.

10 Ohama T, Hori M, Sato K, Ozaki H, Karaki H Chronic treatment with interleukin-1beta attenuates contractions by decreasing the activities of CPI-17 and MYPT- 1 in intestinal smooth muscle. J Biol Chem. 2003;278(49):48794-804.

11 Liebregts T, Adam B, Bredack C, Röth A, Heinzel S, Lester S, et al. Immune activation in patients with irritable bowel syndrome. Gastroenterology. 2007 Mar;132(3):913-20. 
12 Yu YB, Zuo XL, Zhao QJ, Chen FX, Yang J, Dong YY, et al. Brain-derived neurotrophic factor contributes to abdominal pain in irritable bowel syndrome. Gut. 2012;61(5):685-94.

$13 \mathrm{Kim} \mathrm{JH}$, Lin E, Pimentel M. Biomarkers of irritable bowel syndrome. J Neurogastroenterol Motil. 2017 Jan;23(1):20-6.

14 Jones MP, Chey WD, Singh S, Gong H, Shringarpure $\mathrm{R}$, Hoe N, et al. A biomarker panel and psychological morbidity differentiates the irritable bowel syndrome from health and provides novel pathophysiological leads. Aliment Pharmacol Ther. 2014;39(4):426-37.

15 Müller S, Styner M, Seibold-Schmid B, Flogerzi B, Mähler M, Konrad A, et al. Anti-Saccharomyces cerevisiae antibody titers are stable over time in Crohn's patients and are not inducible in murine models of colitis. World J Gastroenterol. 2005;11(44):6988-94.

16 Meijer MJ, Mieremet-Ooms MA, van Hogezand RA, Lamers CB, Hommes DW, Verspaget HW. Role of matrix metalloproteinase, tissue inhibitor of metalloproteinase and tumor necrosis factor-alpha single nucleotide gene polymorphisms in inflammatory bowel disease. World J Gastroenterol. 2007;13(21):2960-6.

17 Kapsoritakis NA, Kapsoritaki IA, Davidi PI, Lotis VD, Manolakis AC, Mylonis PI, et al. Imbalance of tissue inhibitors of metalloproteinases (TIMP) -1 and -4 serum levels, in patients with inflammatory bowel disease. BMC Gastroenterol. 2008;8:55.

18 Nakov R. New markers in ulcerative colitis Clin Chim Acta. 2019 Oct;497:141-6.

19 Buhner S, Hahne H, Hartwig K, Li Q, Vignali $\mathrm{S}$, Ostertag $\mathrm{D}$, et al. Protease signaling through protease activated receptor 1 mediate nerve activation by mucosal supernatants from irritable bowel syndrome but not from ulcerative colitis patients. PLoS One. 2018;13(3): e0193943.

20 Vergnolle $\mathrm{N}$. The enteric nervous system in inflammation and pain: the role of proteinase-activated receptors. Can J Gastroenterol. 2003;17(10):589-92.

21 Buhner S, Hahne H, Hartwig K, Li Q, Vignali $\mathrm{S}$, Ostertag $\mathrm{D}$, et al. Protease signaling through protease activated receptor 1 mediate nerve activation by mucosal supernatants from irritable bowel syndrome but not from ulcerative colitis patients. PLoS One. 2018;13(3): e0193943.

22 Pimentel M, Morales W, Rezaie A, Marsh E, Lembo A, Mirocha J, et al. Development and validation of a biomarker for diarrhea-predominant irritable bowel syndrome in human subjects. PLoS One. 2015;10(5):e0126438.

23 Mohammad BY, Dohouky LA, Mohammed AA. Prevalence of anti-tissue transglutaminase antibodies in patients with irritable bowel syndrome in Duhok city. J Coloproctol. 2019; 39(4):346-50.

24 Hughes PA, Zola H, Penttila IA, Blackshaw LA, Andrews JM, Krumbiegel D. Immune activation in irritable bowel syndrome: can neuroimmune interactions explain symptoms? Am J Gastroenterol. 2013;108(7):1066-74.
25 Nakov NV, Gerova AV, Nakov VR. Faecal calprotectin is a reliable non-invasive marker for assessment of intestinal inflammation in patients with irritable bowel syndrome. C R Acad Bulg Sci. 2013;66(9):1339-44.

26 Walker MM, Talley NJ, Prabhakar M, Pennaneac'h CJ, Aro P, Ronkainen J, et al. Duodenal mastocytosis, eosinophilia and intraepithelial lymphocytosis as possible disease markers in the irritable bowel syndrome and functional dyspepsia. Aliment Pharmacol Ther. 2009;29(7):765-73.

27 Walker MM, Salehian SS, Murray CE, Rajendran A, Hoare JM, Negus R, et al. Implications of eosinophilia in the normal duodenal biopsy: an association with allergy and functional dyspepsia. Aliment Pharmacol Ther. 2010;31(11):1229-36.

28 Gecse K, Róka R, Ferrier L, Leveque M, Eutamene $\mathrm{H}$, Cartier $\mathrm{C}$, et al. Increased faecal serine protease activity in diarrhoeic IBS patients: a colonic lumenal factor impairing colonic permeability and sensitivity. Gut. 2008; 57(5):591-9.

29 Annahazi A, Gecse K, Dabek M, Ait-Belgnaoui A, Rosztóczy A, Róka R, et al. Fecal proteases from diarrheic-IBS and ulcerative colitis patients exert opposite effect on visceral sensitivity in mice. Pain. 2009;144:209-17.

30 Schoepfer AM, Schaffer T, Seibold-Schmid B, Müller S, Seibold F. Antibodies to flagellin indicate reactivity to bacterial antigens in IBS patients. Neurogastroenterol Motil. 2008; 20(10):1110-8

31 Dlugosz A, Törnblom H, Mohammadian G, Morgan G, Veress B, Edvinsson B, et al. Chlamydia trachomatis antigens in enteroendocrine cells and macrophages of the small bowel in patients with severe irritable bowel syndrome. BMC Gastroenterol. 2010;10:19.

32 Chadwick VS, Chen W, Shu D, Paulus B, Bethwaite $\mathrm{P}$, Tie A, et al. Activation of the mucosal immune system in irritable bowel syndrome. Gastroenterology. 2002;122(7):1778-83.

33 Montalto M, Gallo A, Santoro L, D’Onofrio F, Landolfi R, Gasbarrini A. Role of fecal calprotectin in gastrointestinal disorders. Eur Rev Med Pharmacol Sci. 2013;17(12):1569-82.

34 Waugh N, Cummins E, Royle P, Kandala NB, Shyangdan D, Arasaradnam R, et al. Faecal calprotectin testing for differentiating amongst inflammatory and non-inflammatory bowel diseases: systematic review and economic evaluation. Health Technol Assess. 2013;17(55):xv-xix.

35 Mujagic Z, Tigchelaar EF, Zhernakova A, Ludwig T, Ramiro-Garcia J, Baranska A, et al. A novel biomarker panel for irritable bowel syndrome and the application in the general population. Sci Rep. 2016;6:26420

36 Natarajan N, Pluznick JL. From microbe to man: the role of microbial short chain fatty acid metabolites in host cell biology. Am J Physiol Cell Physiol. 2014;307(11):C979-85.
37 Tana C, Umesaki Y, Imaoka A, Handa T, Kanazawa M, Fukudo S. Altered profiles of intestinal microbiota and organic acids may be the origin of symptoms in irritable bowel syndrome. Neurogastroenterol Motil. 2010; 22(5):512-5.

38 Farup PG, Rudi K, Hestad K. Faecal shortchain fatty acids: a diagnostic biomarker for irritable bowel syndrome? BMC Gastroenterol. 2016;16(1):51.

39 Öhman L, Stridsberg M, Isaksson S, Jerlstad P, Simrén M. Altered levels of fecal chromogranins and secretogranins in IBS: relevance for pathophysiology and symptoms? Am J Gastroenterol. 2012;107(3):440-7.

40 El-Salhy M, Lomholt-Beck B, Hausken T, Chromogranin T. Chromogranin A as a possible tool in the diagnosis of irritable bowel syndrome. Scand J Gastroenterol. 2010; 45(12):1435-9.

41 Camilleri M, Halawi H, Oduyebo I. Biomarkers as a diagnostic tool for irritable bowel syndrome: where are we? Expert Rev Gastroenterol Hepatol. 2017;11(4):303-16.

42 Ahmed I, Greenwood R, Costello Bde L, Ratcliffe NM, Probert CS. An investigation of fecal volatile organic metabolites in irritable bowel syndrome. PLoS One. 2013;8(3):e58204.

43 Ianiro G, Eusebi LH, Black CJ, Gasbarrini A, Cammarota G, Ford AC. Systematic review with meta-analysis: efficacy of faecal microbiota transplantation for the treatment of irritable bowel syndrome. Aliment Pharmacol Ther. 2019 Aug;50(3):240-8.

44 Barbara G, Ianiro G. Faecal microbial transplantation in IBS: ready for prime time? Gut. 2020 May;69(5):795-6.

45 Rodiño-Janeiro BK, Vicario M, Alonso-Cotoner C, Pascua-García R, Santos J. A review of microbiota and irritable bowel syndrome: future in therapies. Adv Ther. 2018 Mar; 35(3):289-310.

46 Su T, Liu R, Lee A, Long Y, Du L, Lai S, et al. Altered intestinal microbiota with increased abundance of Prevotella is associated with high risk of diarrhea-predominant irritable bowel syndrome. Gastroenterol Res Pract. 2018;2018:6961783.

47 Dlugosz A, Nowak P, D'Amato M, Mohammadian Kermani G, Nyström J, Abdurahman $S$, et al. Increased serum levels of lipopolysaccharide and antiflagellin antibodies in patients with diarrhea-predominant irritable bowel syndrome. Neurogastroenterol Motil. 2015 Dec;27(12):1747-54.

48 Jabbar SK, Dolan B, Eklund L, Wising C, Ermund A, Johansson A, et al. Association between Brachyspira and irritable bowel syndrome with diarrhoea. Gut. Forthcoming 2020.

49 Park HJ. Dysregulated microRNA expression in irritable bowel syndrome. J Neurogastroenterol Motil. 2016 Apr;22(2):166-7.

50 Liao XJ, Mao WM, Wang Q, Yang GG, Wu WJ, Shao SX. MicroRNA-24 inhibits serotonin reuptake transporter expression and aggravates irritable bowel syndrome. Biochem Biophys Res Commun. 2016;469(2):288-93. 
51 Zhou Q, Costinean S, Croce CM, Brasier AR, Merwat S, Larson SA, et al. MicroRNA 29 targets nuclear factor- $\mathrm{\kappa B}$-repressing factor and Claudin 1 to increase intestinal permeability. Gastroenterology. 2015;148(1):158-e8.

52 Zhou Q, Souba WW, Croce CM, Verne GN. MicroRNA-29a regulates intestinal membrane permeability in patients with irritable bowel syndrome. Gut. 2010;59(6):775-84.

53 Zhou Q, Yang L, Larson S, Basra S, Merwat S, Tan A, et al. Decreased miR-199 augments visceral pain in patients with IBS through translational upregulation of TRPV1. Gut. 2016;65(5):797-805.

54 Fourie NH, Peace RM, Abey SK, Sherwin LB, Rahim-Williams B, Smyser PA, et al. Elevated circulating miR-150 and miR-342-3p in patients with irritable bowel syndrome. Exp Mol Pathol. 2014;96(3):422-5.
55 Kapeller J, Houghton LA, Mönnikes H, Walstab J, Möller D, Bönisch H, et al. First evidence for an association of a functional variant in the microRNA-510 target site of the serotonin receptor-type $3 \mathrm{E}$ gene with diarrhea predominant irritable bowel syndrome. Hum Mol Genet. 2008;17(19):2967-77.

56 Labus JS, Gupta A, Coveleskie K, Tillisch K, Kilpatrick L, Jarcho J, et al. Sex differences in emotion-related cognitive processes in irritable bowel syndrome and healthy control subjects. Pain. 2013;154(10):2088-99.

57 Hong JY, Kilpatrick LA, Labus JS, Gupta A, Katibian D, Ashe-McNalley C, et al. Sex and disease-related alterations of anterior insula functional connectivity in chronic abdominal pain. J Neurosci. 2014;34(43):14252-9.
58 Lowén MB, Mayer EA, Sjöberg M, Tillisch K, Naliboff B, Labus J, et al. Effect of hypnotherapy and educational intervention on brain response to visceral stimulus in the irritable bowel syndrome. Aliment Pharmacol Ther. 2013;37(12):1184-97.

59 Drossman DA, Ringel Y, Vogt BA, Leserman J, Lin W, Smith JK, et al. Alterations of brain activity associated with resolution of emotional distress and pain in a case of severe irritable bowel syndrome. Gastroenterology. 2003;124(3):754-61.

60 Jones MP, Chey WD, Singh S, Gong H, Shringarpure R, Hoe N, et al. A biomarker panel and psychological morbidity differentiates the irritable bowel syndrome from health and provides novel pathophysiological leads. Aliment Pharmacol Ther. 2014;39(4):426-37. 